\title{
In Search of Platinum Meter Bar for Measurement of Metabolic Tumor Volume in Lymphoma
}

\author{
Andrea Gallamini \\ Department of Research and Clinical Innovation, Antoine Lacassagne Cancer Center, Nice, France
}

See the associated article on page 1096.

$\mathbf{I}_{n}$ the current issue of The Journal of Nuclear Medicine, Barrington and Meignan outline an international project aimed at reaching an expert consensus on possible standardization of the computation of metabolic tumor volume (MTV), providing clinicians with a strong and reproducible biomarker to predict the outcome of lymphoma treatment (1). In the various lymphoma subtypes considered so far, MTV has turned out to be a strong predictive tool whatever the tumor segmentation method used. Although all the studies have invariably concluded that MTV has a strong predictive role in treatment outcome, the reproducibility of results has been substantially limited by several methodologic flaws, such as retrospective designs, small sample sizes, heterogeneous populations, different types of software for SUV thresholding and tumor segmentation, and different methods of subtracting physiologic uptake. Therefore, MTV is still far from being used in daily clinical practice for a risk-adapted therapeutic strategy in precision medicine. The unusually high predictive value of this quantitative PET-derived biomarker across all the published studies is interpreted as typical for a continuous variable such as lactate dehydrogenase or $\beta_{2}$-microglobulin. In fact, Ceriani et al., in reporting the value of MTV and tumor lesion glycolysis for prediction of treatment outcome in primary mediastinal B-cell lymphoma, found that "[The] analysis of [quantitative] PET parameters as a continuous variable showed that a significantly higher risk of progression was associated with an increase of MTV" (2). However, besides the methodologic limitations, another hurdle remaining in MTV reproducibility is all the caveats of quantitative readout of PET/CT images exploiting SUV as an absolute variable. This hurdle has been aptly commented on by Shöder in this way: “...it is unknowable whether the same [total-body MTV] would have been calculated if the same patient, under the same biologic conditions, would have undergone PET imaging on two different scanners" (3).

Clinicians will favor standardization and prospective validation of the "best method" for MTV computing in lymphoma as this powerful prognostic tool, once standardized and applicable in clinical

Received Apr. 15, 2019; revision accepted May 29, 2019.

For correspondence or reprints contact: Andrea Gallamini, Department of Research and Clinical Innovation, Antoine Lacassagne Cancer Center, 33 Rue de Valombrose, 06189 Nice, France.

E-mail: andreagallamini@gmail.com

Published online Jun. 6, 2019.

COPYRIGHT @ 2019 by the Society of Nuclear Medicine and Molecular Imaging. DOI: 10.2967/jnumed.119.229252 practice, could revolutionize the standard of care in several lymphoma subsets. In early-stage Hodgkin lymphoma, for example, Cottereau et al. showed that baseline MTV could identify a smaller subset of patients in the H10 trial treated with standard chemotherapy plus radiotherapy, with an even worse treatment outcome, compared with that of the broader group with adverse prognosis identified by the European Organisation for Research and Treatment of Cancer criteria (4). These results are so important that they could potentially impact the standard of care for early-stage Hodgkin lymphoma, a condition for which efforts are constantly ongoing to assess the feasibility and safety of omitting radiotherapy and, thus, its long-term effects such as morbidity and mortality. A similar scenario but with the opposite aim could be sketched for a risk-adapted frontline therapeutic strategy for follicular lymphoma, as patients with a high MTV at baseline and a high score on the Follicular Lymphoma International Prognostic Index 2 have shown a 5 -y progression-free survival of $20 \%$ with a hazard ratio of 5.0, compared with patients with a low MTV and a low score (5). The predictive value of MTV is less evident in diffuse large B-cell lymphoma (DLBCL) for several reasons (6), but mainly because of the mounting evidence that under the broad category of DLBCL are several diseases harboring different genotypes, heterogeneous clinical behavior, and different responses to immunochemotherapy (7). Nonetheless, a good correlation was preliminarily shown in DLBCL between MTV and cell-free circulating DNA (Ash A. Alizadeh, unpublished data, September 2018). Different from tumor burden assessment with traditional radiologic tools, MTV assessment with functional imaging using ${ }^{18}$ F-FDG PET/CT allows for correlation with lymphoma physiopathology and clinical behavior. MTV, in fact, does not merely represent tumor burden but also recapitulates the reactivity of host immunity against the tumor. Examples include Hodgkin lymphoma, in which host immunoreactive cells in the microenvironment account for most of the cellularity in the tumor sample (8), and non-small cell lung cancer, in which PD-1-positive tumor-infiltrating lymphocytes show high glycolytic activity and a great affinity for ${ }^{18}$ F-FDG (9). MTV combined with other biomarkers, such as tumor dissemination assessed by a radiomics technique, superseded MTV alone in predicting treatment outcome in DLBCL (10).

All these obstacles were debated at the Paris meeting cited by Barrington and Meignan (1), with the meeting participants agreeing on the following road map toward reaching international agreement on a tumor-segmentation and MTV-computation procedure for lymphoma.

The first step will be to collect a fixed number of PET/CT baseline staging images for Hodgkin lymphoma, follicular lymphoma, and DLBCL treated with standard therapy and fulfilling the prerequisite for PET scanning, according to the European Association of Nuclear Medicine guidelines. The images will then be uploaded to the study website and sent to the core lab of the study. 
Second, in the core lab, the images will first be checked for compliance with standard quality and then distributed to a panel of nuclear medicine experts representing different cooperative groups, who will delineate the MTV according to their experience and using their preferred software. A consensus workshop will be held afterward to discuss, case by case, the criteria for MTV computing in nodal and extranodal areas. This discussion will include rules for region preselection based on SUV threshold and cluster threshold-volumes, removal of physiologic uptake, removal or addition of regions using easy-to-define criteria, and special criteria for assessing the MTV in bone marrow and spleen.

Third, the final results of the segmentation consensus, or the "platinum-standard MTV," obtained by consensus agreement for both the training set and the validation set will be kept in the core lab and not disclosed. Both the images and the platinum-standard MTV for the training set will be distributed on demand to guest institutions that agree to participate in the project, whereas the platinum-standard MTVs calculated in the validation set will be not disclosed.

Fourth, the medical imaging experts of the guest institutions will compute the MTVs for the training set, with open access to the platinum-standard MTV, thus having the chance to verify and tune their segmentation approach.

Finally, they will compute the MTVs for the validation set and post their segmentation results to the core lab, which will assess them and provide a final report to the guest institutions.

This road map may look cumbersome at first glance, but the search for a standard reference (the so-called platinum standard) for measuring MTV is compelling and can no longer be delayed.

\section{DISCLOSURE}

No potential conflict of interest relevant to this article was reported.

\section{ACKNOWLEDGMENTS}

I kindly thank Dr. Irène Buvat (UMR 1023 INSERM/CEA/ Université Paris Sud, ERL 9218 CNRS) and Stéphane Chauvie (Medical Physics Department, Azienda Ospedaliera S. Croce e Carle, Cuneo, Italy) for technical advice.

\section{REFERENCES}

1. Barrington SF, Meignan M. Time to prepare for risk adaptation in lymphoma by standardizing measurement of metabolic tumor burden. J Nucl Med. 2019;60: 1096-1102.

2. Ceriani L, Martelli M, Zinzani PL, et al. Utility of baseline ${ }^{18}$ FDG-PET/CT functional parameters in defining prognosis in primary mediastinal (thymic) large B-cell lymphoma. Blood. 2015;126:950-956.

3. Schöder M. Metabolic tumor volume in lymphoma: hype or hope? J Clin Oncol. 2016;34:3591-3594.

4. Cottereau AS, Versari A, Loft A, et al. Prognostic value of baseline metabolic tumor volume in early stage Hodgkin lymphoma in the standard arm of the H10 trial. Blood. 2018;131:1456-1463.

5. Meignan M, Cottereau SA, Versari A, et al. Baseline metabolic tumor volume predicts outcome in high-tumor burden follicular lymphoma: a pooled analysis of three multicenter studies. J Clin Oncol. 2016;34:3618-3626.

6. Cottereau AS, Buvat I, Kanoun S. Is there an optimal method for measuring baseline metabolic tumor volume in diffuse large B cell lymphoma? Eur J Nucl Med Mol Imaging. 2018;45:1463-1464.

7. Schmitz R, Wright GW, Huang DW, et al. Genetics and pathogenesis of diffuse large B-cell lymphoma. N Engl J Med. 2018;378:1396-1407.

8. Küppers R, Engert A, Hansmann ML. Hodgkin lymphoma. J Clin Invest. 2012;122: 3439-3447.

9. Lopci E, Toschi L, Grizzi F, et al. Correlation of metabolic information on FDGPET with tissue expression of immune markers in patients with non-small cell lung cancer (NSCLC) who are candidates for upfront surgery. Eur J Nucl Med Mol Imaging. 2016;43:1954-1961.

10. Cottereau AS, Nioche C, Dirand AS, et al. ${ }^{18}$ F-FDG PET dissemination features in diffuse large B-cell lymphoma are prognostic of outcome. J Nucl Med. In press. 